\title{
Single Trial Recognition of Error-Related Potentials During Observation of Robot Operation
}

\author{
Iñaki Iturrate, Luis Montesano, Javier Minguez
}

\begin{abstract}
Recent works suggest that several human cognitive processes elicited during the observation and monitoring of tasks developed by others can be detected in real time. These works have also demonstrated that human brain activity can be used to recover from machine errors, and as reward signals to teach a simulated robot how to perform given tasks. This paper studies the elicitation of this activity during the operation of a real robot. Experimental results have been obtained with 4 participants observing the operation of a 5 d.o.f. robotic arm performing correct/incorrect reaching tasks, while an EEG system recorded their brain activity. The results give evidence that the brain areas that play a role in detection and monitoring of errors also play a role when observing the operation of a real robot, that a brain discriminative response is elicited during the observation of a correct/incorrect operation of a real robot, and that it is possible to learn a classifier that provides online categorization with high accuracy $(80 \%)$.
\end{abstract}

\section{INTRODUCTION}

Event-Related Potentials (ERP) are signals that are elicited by the presence of an internal or external event [1], usually recorded by means of an electroencephalogram (EEG). In cognitive neuroscience, it is well known the usage of the ERP to study the underlying mechanisms of human error processing, sometimes referred to Error-related Potentials (ErrPs) [2]. This is because the observation/execution of an incorrect action for the user triggers an activity or potential. This potential codifies the difference between the user's expected outcome and the actual one. Different ErrPs have been described, for instance, when a subject performs a choice reaction task under time pressure and realizes that he/she has committed an error [3] (response ErrPs); when the subject perceives an error committed by another person (observation ErrPs) [4]; when the subject delivers an order and the machine executes another one [2] (interaction ErrP); and recently when the subject perceives an error committed by a simulated robot [5].

Recent studies have shown that it is possible to use these error potentials in a Brain-Computer Interface context. In [2], the authors demonstrated the feasibility of detecting these potentials online, and proposed their use to recover from BCI errors when operating a wheelchair controlled by asynchronous EEG activity. In a similar way, in [6], the authors proposed the use of single-trial detection of

Iñaki Iturrate, Luis Montesano and Javier Minguez are with the Instituto de Investigación en Ingeniería de Aragón (I3A) and Dpto. de Informática e Ingeniería de Sistemas (DIIS), Universidad de Zaragoza, Spain. Email: iturrate@unizar.es, montesano@unizar.es and jminguez@unizar.es. This work has been partially supported by projects HYPER-CSD200900067, DPI2009-14732-C02-01 funded by the Spanish Government and the Portuguese FCT project PTDC/EEA-ACR/70174/2006 error potentials to detect misinterpreted commands in a P300-based speller. Finally, in [5], the authors proposed the detection and use of these signals as a reward for a simple Reinforcement Learning task. However, the question is whether this framework is potentially usable in a real robotic context (e.g. a rehabilitation prosthesis), that is, whether the error mechanisms of the brain are also elicited by observing a real robot operation, and the feasibility of detecting these signals in real-time. The benefits of this framework would be the possibility to detect online an incorrect operation of the robotic device and correct its behavior.

To study this question, this paper presents an experiment developed with a real robot. Experimental results have been obtained with 4 participants observing the operation of a 5 degrees of freedom (d.o.f.) robotic arm performing correct/incorrect reaching tasks, while an EEG system recorded their brain activity. The results suggest that: (a) the brain areas that play a role in detection and monitoring of errors also play a role when observing the operation of a real robot; (b) a brain discriminative response is elicited during the observation of a correct/incorrect operation of a real robot, (c) this response is consistent among different subjects, (d) it is possible to learn a classifier that provides online categorization with high accuracy $(\sim 80 \%)$.

\section{PRotocol AND Design OF THE EXPERIMENT}

This section describes the design of the main experiment of the paper. The objective is to collect the EEG to determine: (a) if a specific brain potential is elicited during the observation of a correct/incorrect operation of a real robot, and if this response is consistent among different subjects; and (b) if it is possible to learn a classifier that provides online categorization with enough accuracy, to evaluate the feasibility of an online Brain-Computer Interface.

In the experiment, it was used a Katana300 robot arm with 5 degrees of freedom. The instrumentation used to record the EEG brain activity was a gTec system. The location of the electrodes was selected following previous ErrP studies [5] at FP1, FP2, F7, F8, F3, F4, T7, T8, C3, C4, P7, P8, P3, P4, O1, O2, AF3, AF4, FC5, FC6, FC1, FC2, CP5, $\mathrm{CP} 6, \mathrm{CP} 1, \mathrm{CP} 2, \mathrm{Fz}, \mathrm{FCz}, \mathrm{Cz}, \mathrm{CPz}, \mathrm{Pz}$ and $\mathrm{Oz}$ (according to the international $10 / 10$ system). The ground electrode was positioned on $\mathrm{FPz}$ and the reference electrode was placed on the right earlobe. The EEG was amplified, digitized with a sampling frequency of $256 \mathrm{~Hz}$, power-line notch-filtered, and $0.5-10 \mathrm{~Hz}$ bandpass-filtered. Additionally, a Common Average Reference (CAR) Filter was applied to remove any background activity detected on the signal. The signal 


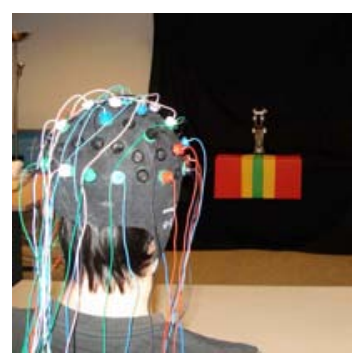

(a)

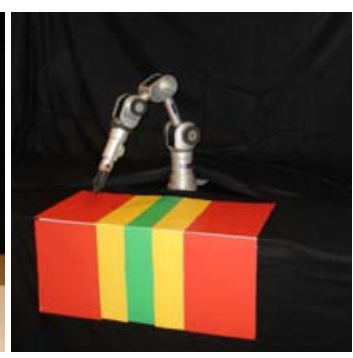

(b)
Fig. 1. (a) General view of the setup. The subject observes the robotic arm motion while the EEG system records the brain activity. (b) The robot arm performs consecutive reaching tasks to five predefined positions.

recording and processing and the synchronization between the robot arm and the EEG were developed under BCI2000 platform [7]. The general setting of the experiment was a user observing the operation of a robot arm while the EEG was recorded (Figure 1a). The robot continuously operated by developing reaching tasks to five predefined positions (Figure 1b). The participants were instructed to judge the robot motion as follows: $(a)$ a motion towards the center was a correct operation, $(b)$ a motion towards the locations placed just on the side (left or right) of the center was a small operation error, and $(c)$ a motion towards the furthest locations from the center (left or right) was a large operation error. The reaching positions were marked with colors to facilitate the participants the identification of the operations, where green was the correct movement, yellow the small operation errors, and red the large operation errors.

Four male, right-handed, 24-aged people participated in the experiments. The participants were informed about the experiment. They were instructed to avoid as much as possible any muscular movement to avoid the contamination of the EEG (artifacts), especially the lateral eye movements. The protocol was adapted to minimize the motion of the eyes by placing the robot arm far enough from the subject (4 meters).

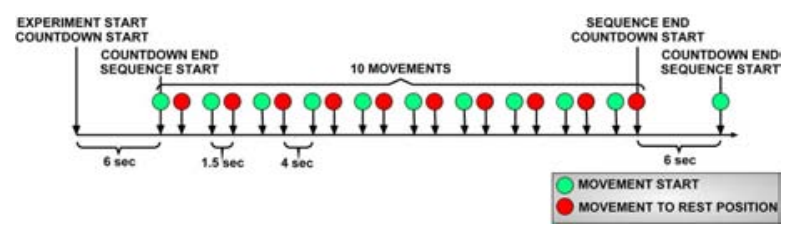

Fig. 2. Temporal diagram of a sequence of robot actions.

For each participant, an experiment consisted of 10 trials of 5 sequences each, where each sequence was composed by 10 random reaching actions carried out by the robot arm. A total number of 500 operations were executed. Each sequence was designed as follows (Figure 2): firstly there was a 6 seconds countdown with auditory signals associated (to inform the participant that the sequence was starting) and then ten random actions were executed by the robot. The reaching action lasted for $1.5 \mathrm{~s}$, and was composed by two phases: the motion to the actual location, which lasted for $0.8-1.1 \mathrm{~s}$ depending on the action, and a second phase where
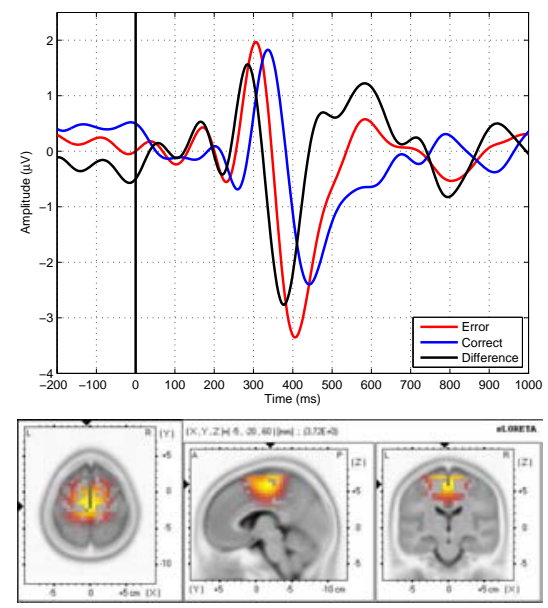

Fig. 3. Time-locked average waveforms (up) and SL at $375 \mathrm{~ms}$ (down) averaged over all the participants in channel Cz. A baseline of $200 \mathrm{~ms}$ before the movement started is also shown. The SL figure is better understood in color, where yellow and red areas indicate a high brain activity.

the robot stayed on the final position without moving (which lasted for $0.4-0.7 \mathrm{~s}$ depending on the action). The returning to the rest position lasted for $4 \mathrm{~s}$. In a similar way, it had two phases: the first one was the returning motion (which lasted for $0.8-1.1 \mathrm{~s}$ ), and a second phase where the robot stayed on the rest position for $2.9-3.2 \mathrm{~s}$, providing the participants some time to relax between robot motions. The total time of the experiment was 50 minutes plus approximately 5 minutes of breaks distributed between trials.

The experiment was designed in such a way that the 500 operations were equally distributed as 100 times per possible action. Thus, 100 ERP brain responses of each action were recorded.

\section{NEUROPHYSIOLOGYICAL ANALYSIS}

After recording the EEG data, the first step was to characterize the brain response as a possible ERP. Previous to the analysis, possible eye blinking artifacts were removed using a threshold-based method. The analysis was developed as follows. Firstly, the averaged ERP potentials for each participant were constructed, which are simply the averaged sum of the individual responses for each condition at each electrode, in order to improve the signal-to-noise ratio and, as a consequence, filter background noise and occasional artifacts. This averaged ERP was then averaged for the four participants for error versus correct responses. Additionally, in order to speculate about the brain areas involved in the generation of the potentials, we used sLORETA [8], an EEG Source Localization (SL) technique. This type of techniques estimates the neural generators within the brain given the EEG at the surface of the scalp.

Figure 3 shows the results of the averaged ERPs in the $\mathrm{Cz}$ electrode (usually selected to display error-related potentials), and the result of the source localization technique.

The first observation is that the averaged ERPs resulting from the robot correct/incorrect operations are different, which implies that on average, there are different brain 
processes involved. Secondly, the shape of the response in $\mathrm{Cz}$ elicited by the incorrect operations, and by the difference signal (error minus correct responses on average) are similar to the responses of other protocols that involve the human monitoring of errors, concretely the interaction errors (see [9] for some examples): they have a sharp positive potential at around 0.3 seconds, followed by a wide prominent negativity around 0.4 seconds. Thirdly, the main active areas at the time of the prominent negativity of the difference signal ( $~ 375 \mathrm{~ms}$ ) were Brodmann Areas (BA) 6, 31, 5, 24 and 4 (Figure 3). These activations conform a brain activity on the frontier of the Pre-Supplementary Motor Area (PreSMA, BA 6 and 4), Anterior Cingulate Cortex (ACC, BA 24) and Posterior Cingulate Cortex (PCC, BA 31 and 5). As discussed on [9], the results suggest the existence of an activity related with error detection and processing (due to ACC and Pre-SMA), as well as a more posterior activity, which could be related with spatial attention [10] (due to PCC). The error-related areas also agree with several results that obtained the same areas in the most prominent negativity in reaction, observation and interaction errors [9], [3], [4]. These results support the hypothesis that a discriminative (correct/incorrect) Event-Related Potential is elicited during the human monitoring of the robot operation.

\section{CALIBRATION AND REAL-TIME ClASSIFICATION}

The objective of this section is to perform a single trial classification of the ERPs. We studied two classification tasks: error versus correct responses and small versus large errors.

The classification process is composed of two different phases: feature extraction and classification. The used features were the RAW data subsampled to $64 \mathrm{~Hz}$. In order to select which features use (i.e, which channels and time window), a statistical measure that shows the areas significantly different between error and correct responses was performed. Concretely, we computed the $r^{2}$ analysis [7], widely used in neurophysiology for this purpose. Figure 4a shows the $r^{2}$ for error versus correct responses, for every channel within the time window $0-800 \mathrm{~ms}$, averaged for all the participants. The activity on the ERP is clearly centered on the $\mathrm{FCz}$ and $\mathrm{Cz}$ electrodes, suggesting a fronto-central activity and thus agreeing with the analysis performed on the previous section. Despite there is a significantly different activity on other channels, these could be related with other cognitive processes and therefore not related with error processing. Thus, we selected (by visual inspection) for classification the following fronto-central channels: $\mathrm{Fz}, \mathrm{FC} 1, \mathrm{FC} 2, \mathrm{FCz}, \mathrm{Cz}$, $\mathrm{CP} 1, \mathrm{CP} 2$ and CPz. ${ }^{1}$ Finally, the time window was also fixed by visual inspection according to the $r^{2}$ results (see footnote 1), selecting the range $200-800 \mathrm{~ms}$. This selection leaded to a feature vector (concatenating all the channels selected), of

\footnotetext{
${ }^{1}$ Notice that we are aware of a possible effect of overfitting because of using all the examples to select the channels and the time window. For this reason, we also computed the $r^{2}$ separately for each participant and also with different percentages of the data, having no substantial differences on the results obtained.
}

312 features. Regarding the small vs large errors case, the $r^{2}$ within the time window $0-1000 \mathrm{~ms}$ is shown on Figure $4 \mathrm{~b}$. For this second classification task, the differences are clearly later when compared with the first task. This is due to the fact that the movement of the robot was continuous, and thus the participants noticed the differences between these actions later. In this case, we chose the range $700-900 \mathrm{~ms}$, having a total of 104 features.

The previous features were normalized on the range $0-1$, and they were used to train a Support Vector Machine (SVM). This classifier has been used on the past for classifying error-related potentials [6], [11]. Among all the possible versions of SVM, we used the $\nu$-SVM classifier with a radial basis function $\mathrm{kernel}^{2}$. The $\nu$ parameter was set to 0.5 and the $\gamma$ parameter of the radial basis function was set to $\frac{1}{\sharp \text { features }}$.

For the error/correct classification task, we selected $25 \%$ of each type of incorrect movement so as to have balanced data, thus having 100 error examples and 100 correct examples. For the second classification task, we had 200 small errors and 200 large errors. Finally, in order to minimize overfitting effects, we used a ten-fold cross-validation strategy to train the classifier. Furthermore, the normalization values were calculated with the $90 \%$ of the data and applied to the remaining $10 \%$.

The classification performances (True Positives and True Negatives) for the error/correct classification task for each participant are shown on table I.

TABLE I

CLASSIFIER PERFORMANCE: ERROR VS CORRECT RESPONSES

\begin{tabular}{l|cccc|c} 
& $\mathrm{P} 1$ & $\mathrm{P} 2$ & $\mathrm{P} 3$ & $\mathrm{P} 4$ & Average \\
\hline Error & $84.67 \%$ & $77.56 \%$ & $76.00 \%$ & $77.67 \%$ & $\mathbf{7 8 . 9 7 \%}$ \\
Correct & $90.56 \%$ & $80.78 \%$ & $80.00 \%$ & $79.00 \%$ & $\mathbf{8 2 . 5 8} \%$
\end{tabular}

The results show a high detection rate of the ERPs, being roughly an $80 \%$ on average. The accuracies are always higher on the correct responses. In general, these results demonstrate the feasibility of detecting these signals on single trial when elicitated by a robotic arm. However, for the small/large errors classification task, we obtained on average a $57.80 \%$ and $60 \%$ of accuracy for small and large errors respectively. These results suggest that, despite there were differences between the small and large errors (see Figure 4b), they were rather subtle to obtain good performances.

Additionally, when using Brain-Computer Interfaces for real applications, it is important to know the amount of data needed for training in order to achieve good detection rates (the EEG data acquisition is a consuming and tiring process). Thus, we studied the evolution of the classification rate as a function of the number of training examples, for error versus correct responses. The analysis was performed selecting sets as follows: using the first (in time) $10 \%$ of the data as training set and the $90 \%$ of the last (in time) data as the test set (labeled as 10\%-90\%). We performed this comparison

\footnotetext{
${ }^{2}$ We made a comparison among different linear and non-linear kernels for SVM, obtaining slightly better results with the radial basis function kernel.
} 


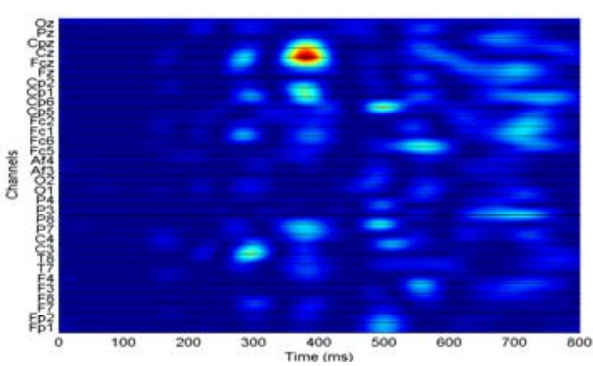

(a)

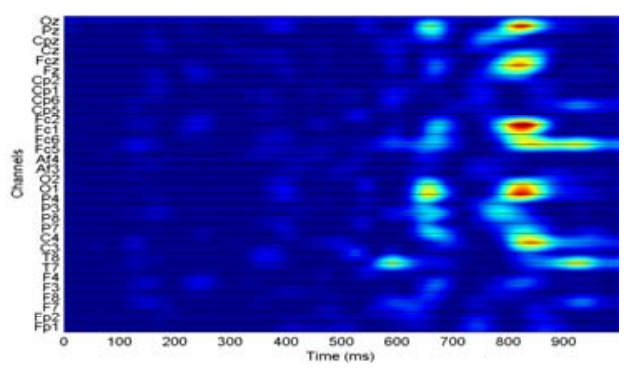

(b)

Fig. 4. (a) $r^{2}$ of the Error vs Correct Responses (time versus channels). Notice that it is only shown the window $0-800$ ms, since the error ERP is elicited within this range. (b) $r^{2}$ of the Small vs Large error responses. In this case the differences start later since the movement is continuous, and thus the $0-1000 \mathrm{~ms}$ is shown. The figure is best viewed in color.

also for the cases $20 \%-80 \%, 30 \%-70 \%, 40 \%-60 \%, 50 \%$ $50 \%, 60 \%-40 \%, 70 \%-30 \%, 80 \%-20 \%$ and $90 \%-10 \%$. Figure 5 shows the recognition rate for each class averaged over the four participants.

The results show that the recognition rate reached a stable value with a $60 \%$ of the data (60 examples of each action), equivalent to around 35 minutes of data collection.

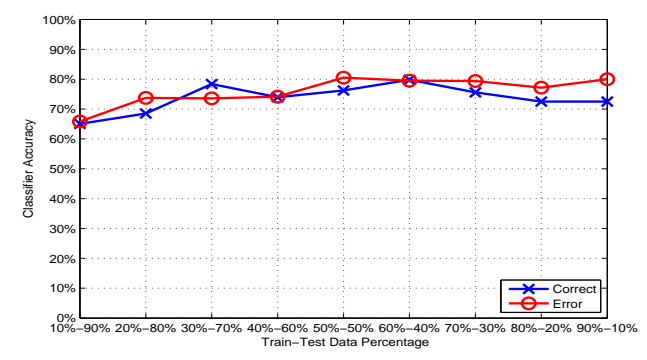

Fig. 5. Classification accuracies for different percentages of training and test data.

Notice that these results also suggest that the protocol for further experiments does not require such a long training as the one used in this paper. For instance, the $60 \%-40 \%$ case represent a first phase of training, of roughly 30 minutes, and a second phase concerning the experiment as such, where the signal is detected online using the previous training data.

Summarizing, we have shown that it is possible to distinguish between error and correct robot operations with a high accuracy $(\sim 80 \%)$. In the case of the percentages analysis, a data collection of 30 minutes with 60 examples is required to obtain stable classification rates.

\section{CONCLUSIONS AND FUTURE WORK}

In this paper, we have demonstrated the existence of a brain response during the observation of a real robot action. The results show that the brain areas involved in this brain activity are those related with prior work on human error processing. The nature of this response, together with the ability to classify single-trial EEG measurements, opens the door to develop prostheses that could learn users' desires and adapt to time-specific requirements.

Our future work focuses on extract more information on the responses obtained. Concretely, the differentiation be- tween various aspects of errors, such as laterality (left-right) and degree (small-large), may play an important role when being in more complex settings, such as continuous domains, and therefore needs more thorough studies. Furthermore, we are also studying selection methods that automatically selects the time window and channels according to the neural process of interest. We also plan to explore the detection of errors on a continuous EEG signal to incorporate this on more complex robot actions. In the long term, the objective of this work would be to online detect these signals on real rehabilitation applications, such as robotic prostheses, so as to correct the device's behavior.

\section{REFERENCES}

[1] J.R.Wolpaw, N.Birbaumer, D.J.McFarland, G.Pfurtscheller, and T.M.Vaughan, "Brain-computer interfaces for communication and control," Clinical Neurophysiology, vol. 113, no. 6, pp. 767-91, June 2002.

[2] P.W. Ferrez and J.d.R Millán, "Simultaneous real-time detection of motor imagery and error-related potentials for improved BCI accuracy," in Proceedings of the 4th International Brain-Computer Interface Workshop \& Training Course, 2008, pp. 197-202.

[3] M. Falkenstein, J. Hoormann, S. Christ, and J. Hohnsbein, "ERP components on reaction errors and their functional significance: A tutorial," Biological Psychology, vol. 51, pp. 87-107, 2000.

[4] H.T. van Schie, R.B. Mars, M.G.H Coles, and H. Bekkering, "Modulation of activity in medial frontal and motor cortices during error observation," Neural Networks, vol. 7, pp. 549-554, 2004.

[5] I. Iturrate, L. Montesano, and J. Minguez, "Robot Reinforcement Learning using EEG-based reward signals," in IEEE International Conference on Robotics and Automation (ICRA), 2010.

[6] G. Visconti, B. Dal Seno, M. Matteucci, and L. Mainardi, "Automatic recognition of error potentials in a P300-based brain-computer interface," in Proceedings of the 4th International Brain-Computer Interface Workshop \& Training Course, 2008, pp. 238-243.

[7] G. Schalk, D.J. McFarland, T. Hinterberger, N. Birbaumer, and J.R. Wolpaw, "BCI2000: A General-Purpose Brain-Computer Interface (BCI) System," IEEE Transactions on Biomedical Engineering, vol. 51, no. 6, May 2004.

[8] R.D. Pascual-Marqui, "Standardized low resolution brain electromagnetic tomography (sLORETA): Technical details.," Methods and Findings in Experimental and Clinical Pharmacology, pp. 5-12, 2002.

[9] P.W. Ferrez, Error-Related EEG Potentials in Brain-Computer Interfaces, Ph.D. thesis, École Polytechnique Fédérale de Laussane, 2007.

[10] Y. Kim, D.R. Gitelman, A.C. Nobre, T.B. Parrish, K.S. LaBar, and M. Mesulam, "The Large-Scale Neural Network for Spatial Attention Displays Multifunctional Overlap But Differential Asymmetry," Neurolmage, vol. 9, pp. 269-277, 1999.

[11] F. Lotte, M. Congedo, A. Lécuyer, F. Lamarche, and B. Arnaldi, "A review of classification algorithms for EEG-based brain-computer interfaces," Journal of Neural Engineering, vol. 4, pp. R1, 2007. 